\title{
COVID-19 and hypercoagulability
}

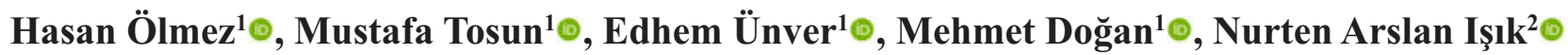

${ }^{1}$ Department of Pulmonary Diseases, Erzincan Binali Yıldırım University School of Medicine, Erzincan, Turkey

${ }^{2}$ Department of Child Development, Erzincan Binali Yıldırım University Faculty of Health Sciences, Erzincan, Turkey

\begin{abstract}
It has been observed that patients with COVID-19 infection may develop acute pulmonary embolism, acute myocardial infarction, limb thrombosis, and venous and/or arterial thrombosis, including central nervous system. Thrombosis formation in COVID-19 patients can be explained by the virchow triad. Severe Acute Respiratory Syndrome-Coronavirus-2 (SARS-CoV-2) can directly attack vascular endothelial cells, causing excessive activation of the immune system and cytokine storm, causing thrombosis. Increased prothrombotic factors such as antiphospholipid antibodies, elevated factor VIII, high fibrinogen, circulating prothrombotic microparticles, neutrophil extracellular traps have been reported in COVID-19 infection. It has been argued that complement-mediated endothelial damage, increase in pro-inflammatory cytokines such as interleukin (IL)-1, IL-6, IL-8 and interferon- $\gamma$ may be the cause of thrombosis. Autopsies of patients with COVID-19 revealed that the causes of death were pneumonia and pulmonary embolism. When monitoring COVID-19 patients, platelet, prothrombin time (PT) and activated partial thromboplastin time (aPTT), fibrinogen and Ddimer monitoring should be initiated every 1-2 days, especially in critically ill patients. High D-dimer levels are associated with high mortality; may indicate infection/sepsis, cytokine storm, and impending organ failure. Disseminated intravascular coagulation (DIC) may be seen in COVID-19 patients, but unlike DIC, fibrinogen is usually high. Clotting times and platelet counts are usually normal. Therefore, it is appropriate to use sepsisinduced coagulopathy (SIC) criteria in the follow-up of COVID-19 patients. Infected areas related to pulmonary embolism can be seen as radiological appearance. Some patients may have enlarged subsegmental pulmonary vessels. Treatment of the underlying disease is the most important treatment for all coagulopathies. Patients with venous thromboembolism, inpatient medical, surgical, and COVID-19 therapy should receive anticoagulant therapy unless there is a contraindication to anticoagulation (for example, active bleeding or severe bleeding within the previous 24 to 48 hours).
\end{abstract}

Keywords: COVID-19, hypercoagulability, severe acute respiratory syndrome

$\mathrm{C}$ oronavirus disease-19 (COVID-19) caused by Severe Acute Respiratory Syndrome-Coronavirus-2 (SARS-CoV-2) is a potentially fatal disease. Many mechanisms can be involved in mortality. One of them is disseminated intravascular coagulation (DIC) caused by coagulopathy caused by SARS-CoV-
2 [1-3]. This condition has been called thromboinflammation or COVID-19-associated coagulopathy by some experts $[4,5]$.

There are few studies on the state of hypercoagulability caused by COVID-19. Experience comes mostly from research on SARS, Middle East respira- 
tory syndrome (MERS), and the influenza virus. Studies have shown that severe COVID-19 patients are usually complicated with coagulopathy and most of the deaths have DIC [1]. It has been observed that patients with COVID-19 infection may develop venous and/or arterial thrombosis, including acute pulmonary embolism (PE), acute myocardial infarction, extremity thrombosis, and central nervous system [6].

\section{PHYSIOPATHOLOGY}

It has been suggested that the pathophysiology of akut PE is different from classical pulmonary thromboembolism and that thrombosis occurs locally in the lung vessels due to a local inflammatory process instead of the classical embolism from another part of the body [7]. Since the pathophysiology of thrombotic disease is different, it may be questioned whether the akut PE standard therapy (prophylactic or therapeutic) with low-molecular-weight heparin (LMWH), direct oral anticoagulants or vitamin $\mathrm{K}$ antagonist is sufficient. Thrombosis formation in COVID-19 patients can be explained by the virchow triad. SARS-CoV-2 protein, which causes viral pneumonia and inflammatory storm (cytokine storm) and DIC, infects human cells through angiotensin-converting enzyme 2 (ACE2). ACE2 is found in alveolar epithelial cells, arterial endothelial cells, small intestine epithelial cells, and immune tissues [8]. SARS-CoV-2 can activate the coagulation system by directly attacking vascular endothelial cells. SARS-CoV-2 can also activate the natural immune system after it enters the body. Overactivation of the immune system can cause cytokine storms, damage the microvascular system, activate the coagulation system while inhibiting fibrinolysis and anticoagulation systems. The RAAS pathway is activated by binding the virus to ACE2 and causing a decrease in enzyme expression. RAAS activation; With thrombocyte adhesion and aggregation, there is a theoretical risk of pulmonary embolism, pulmonary hypertension and fibrosis. It is known that the coagulation pathway can be activated by the contact system and the kallikrein/kinin system (KKS) [9]. Since KKS SARS-CoV-2 is dysregulated by the binding of type II pneumocytes to the ACE2 receptor, this may be a reasonable mechanism for the stated interaction between COVID-19 and pulmonary vessel thrombosis [10]. Prothrombotic factors such as circulating antiphospholipid antibodies and elevated factor VIII, high fibrinogen, circulating prothrombotic microparticles, neutrophil extracellular traps (NETs) have been reported in patients with severe COVID-19 $[11,12]$.

Antiphospholipid antibodies that can prolong activated partial thromboplastin time are common in viral infections. However, it is usually temporary and does not always mean that the risk of thrombosis increases $[13,14]$. The induction of antiphospholipid syndrome (APS) by viral infection can be explained by a series of endothelial damage, platelet activation and thrombosis formation mediated by antiphospholipid antibodies. APS can present as catastrophic APS in many patients. Catastrophic APS and DIC are clinically similar and differential diagnosis can be difficult. APS manifests itself with more hypercoagulability tendency. Diagnosis depends mainly on the measurement of antiphospholipid antibodies.

Interleukin (IL)-6 is an important factor involved in the cytokine storm induced by SARS-CoV-2. IL-6 stimulates the liver to produce more thrombopoetin and fibrinogen [15]. Complement-mediated endothelial damage may also contribute to hypercoagulability [16]. Most cases of sepsis COVID-19 pneumonia meet the Sepsis Third International Consensus Criteria (sepsis-3) [17]. Besides endothelial damage with complement activation seen in sepsis, inflammatory and microthrombotic pathway activation predisposes to thrombosis and leads to DIC. The dysfunction of endothelial cells induced by infection results in excessive thrombin production and inhibition of fibrinolysis. This causes hypercoagulability in the infected patient, such as COVID-19 [18, 19]. Complement activation not only causes direct endothelial damage, but also involves leukocytes with the formation of $\mathrm{C} 3 \mathrm{a}$ and $\mathrm{C} 5 \mathrm{a}$, which are responsible for the local release of pro-inflammatory cytokines such as interleukin IL-1, IL-6, IL-8 and interferon [20]. The resulting excessive host immune response lymphocytes, resident macrophages, monocytes and neutrophils cause strong pro-inflammatory response, causing severe collateral tissue damage, excessive vascular endothelial and alveolar epithelial cell damage. In addition, they cause microvascular thrombosis [21, 22]. In the late stages of acute respiratory distress syndrome (ARDS), the pro- 
gression of endothelial damage with microvascular thrombosis may spread from the lung and affect the microvascular bed of the kidneys, brain, and other vital organs [23]. This progressive state may involve alveolar macrophage, complement activation lectin, the brain and other vital organs, and may contribute to multiple organ failure. This condition is called Endothelial Thromboinflammatory Syndrome. In addition, this excessive inflammatory response causes progressive worsening of ventilation / perfusion imbalances, loss of hypoxic vasoconstriction reflexes, and microvascular pulmonary thrombosis, in addition to the functional effects of ARDS pathogenesis [24]. In addition, hypoxia observed in severe cases of COVID-19 not only increases blood viscosity, but also stimulates thrombosis through a signaling pathway dependent on hypoxia-induced transcription factors (HIF) [25].

\section{COVID-19 AUTOPSY CASES}

Autopsy studies of patients with COVID-19 revealed that the common causes of death were pneumonia and PE $[16,26]$. In another recently published autopsy study, vascular congestion and edema in the alveolar septum, mononuclear and lymphocyte infiltration, thrombus in small vessels and capillaries, vascular endothelial and intimal damage in the cardiovascular system, and microthrombus in the liver portal region have been demonstrated, which causes COVID-19 to thrombosis in many organs. show that it can cause $[27,28]$. In 18 postmortem patients with COVID-19, all cases with deep vein thrombosis (DVT) were reported to have bilateral leg involvement and none of them were suspected before they died. These patients were older, obese male patients with diabetes mellitus and/or cardiovascular disease.

\section{LABORATORY}

While monitoring COVID-19 patients, platelet, prothrombin time (PT) and activated partial thromboplastin time (aPTT), Fibrinogen and D-dimer monitoring should be initiated every 1-2 days with diagnosis in critically ill patients. Table 1 and Table 2 show the laboratory parameters to be monitored in
COVID-19 infection [29].

In a study, it was observed that acute extremity ischemia can develop in patients with COVID-19, especially when D-dimer levels are high [30]. High D-dimer levels at admission are associated with high mortality; may be a marker of infection/sepsis, cytokine storm and impending organ failure [31]. Therefore, hospital admission of patients with significantly higher D-dimer levels should be considered even in the absence of other signs of violence [32]. Schistocytes can be seen in peripheral smears of patients with COVID-19 infection. Increases in lactate dehydrogenase, ferritin, C-reactive protein, D-dimer and Interleukin seen in COVID-19 disease indicate that the disease is pro-inflammatory and prone to hypercoagulability [3]. Other diagnostic tests commonly performed in patients are prothrombin time and platelet count. Thrombocytopenia is generally considered an indicator of sepsis mortality. An increase in thrombocytopenia greater than five times has been associated with an increased risk of severe COVID-19 disease. In addition to the platelet count, PT and D-dimer levels, it may be useful to determine the serum fibrinogen level as recommended in the DIC guidelines of the International Society for Thrombosis and Hemostasis (ISTH). The significant increase in D-dimer, fibrin degradation products in patients in the state of ischemia supported the prediction that multiple microthrombosis occurs in the body. Experience with monitoring PT, D-dimer, platelet count, and fibrinogen levels for septic coagulopathy suggests that it can be helpful in determining the prognosis in patients with hospitalized COVID-19. If these parameters deteriorate, more aggressive critical care support may be required [33-35]. Coagulopathy in COVID-19 patients

\section{Table 1. Laboratory values}

\begin{tabular}{lcc}
\hline Test & \multicolumn{1}{c}{ Result } & Comment \\
\hline Platelets & $<100.000 / \mu \mathrm{L}$ & Sepsis, high mortality \\
& $<150.000 / / \mu \mathrm{L}$ & Severe illness \\
PT & $>3 \mathrm{sec}$ increase & Intensive care patient \\
aPTT & $>5 \mathrm{sec}$ increase & \\
Fibrinogen & $<150 \mathrm{mg} / \mathrm{mn}$ & High mortality \\
D-dimer & $\times 4$ increase & High risk patient \\
\hline $\begin{array}{l}\text { PT }=\text { prothrombin time, aPTT } \\
\text { thromboplastin time }\end{array}$ &
\end{tabular}


Table 2. Evaluation of coagulopathy in COVID-19 infection

\begin{tabular}{lcc}
\hline Respiratory Failure & \multicolumn{2}{c}{ Organ Failure } \\
\hline COVID-19 Infection & pre-DIC & DIC \\
$\begin{array}{l}\text { Fibrinogen, D-Dimer } \\
\text { (partial increase) }\end{array}$ & $\begin{array}{c}\text { D-Dimer, PT (severe } \\
\text { increase) }\end{array}$ & PLT - decrease, \\
\hline
\end{tabular}

$\mathrm{DIC}=$ disseminated intravascular coagulation, $\mathrm{PT}=$ prothrombin time

emerges on an average of 7 days. Decreased antithrombin-3 levels have been observed in patients, but this is very rare and routine monitoring is not recommended. Plasma tissue factor and plasminogen activator inhibitor-1 was found to be higher in patients with ARDS than in patients with no development [36]. The hypercoagulant status associated with COVID-19 meets the DIC criteria published by ISTH in 2009 (Table 3) [29, 33]. However, the most important clinical finding in COVID-19 is thrombosis, and the most important finding in acute decompensated DIC is bleeding. There are many laboratory findings similar to COVID-19 DIC. D-Dimer is significantly increased, mild thrombocytopenia may be seen. However, other coagulation parameters in COVID-19 are different from DIC. Unlike DIC in many COVID-19 patients, fibrinogen is usually elevated and clotting times and platelet counts are generally normal. PT and aPTT are normal or slightly prolonged in patients with

\section{Table 3. ISTH DIC criteria}

\begin{tabular}{lcc}
\hline Variable & Value & Score \\
\hline Thrombocyte $\times 109 / \mathrm{L}$ & $>100$ & 0 \\
& $50-100$ & 1 \\
& $<50$ & 2 \\
\hline D-Dimer $(\mathrm{ng} / \mathrm{mL})$ & $<400$ & 0 \\
& $400-4000$ & 2 \\
\hline Prolonged PT & $>4000$ & 3 \\
\hline & $<3 \mathrm{sn}$ & 0 \\
\hline Fibrinogen $\mathrm{g} / \mathrm{L}$ & $3-6 \mathrm{sn}$ & 1 \\
\hline & $>6 \mathrm{sn}$ & 2 \\
\hline A score & $>1$ & 0 \\
\hline
\end{tabular}

A score of $>5$ suggests DIC. DIC $=$ disseminated intravascular coagulation, ISTH $=$ International Society for Thrombosis and Hemostasis, $\mathrm{PT}=$ prothrombin time
COVID-19 infection, normal or increased platelet count, increased fibrinogen, increased D-Dimer values, Factor VIII activity increased, VWF antigen greatly increased, antithrombin and free protein $\mathrm{S}$ low protein $\mathrm{C}$ was found to be slightly increased. It suggests that the consumption of coagulation factors does not occur in COVID-19 [11]. In contrast, acutely decompensated DIC is associated with low fibrinogen due to consumption of coagulation factors. In one of the large series in which thromboembolic events were reported, none of the patients developed DIC [6].

Typically, bleeding is predominant in acute decompensated DIC. Thrombosis is predominant in chronic compensated DIC. Sometimes both situations can happen together. Therefore, the development of hypercoagulable in patients with COVID-19 is more similar to compensated DIC. However, in COVID-19, the platelet count and aPTT are usually normal. DIC definition of ISTH scoring system is based on laboratory findings and is designed for use only in patients with an underlying condition known to be associated with DIC [33]. A score of 5 or more indicates that DIC is possible. Despite this, DIC is diagnosed clinically. There is no gold standard or specific test in the diagnosis of DIC [33]. ISTH proposed a new category of sepsis-associated DIC, called sepsis-induced coagulopathy (SIC), which defines a stage earlier than DIC (Table 4) [29, 37]. Since the decrease in platelet count and PT prolongation are associated with increased

\section{Table 4. Sepsis induced coagulopathy (SIC)} criteria

\begin{tabular}{lccc}
\hline Score & $\mathbf{0}$ & $\mathbf{1}$ & $\mathbf{2}$ \\
\hline PT & $<1.2$ & $>1.3$ & $>1.4$ \\
Thrombocyte $\times 109 / \mathrm{L}$ & $>150$ & $<150$ & $<100$ \\
Total & 0 & 1 & $>2$ \\
\hline
\end{tabular}

Note: $>4$ points makes $\mathrm{SIC}$ think. $\mathrm{PT}=$ prothrombin time 
mortality, and hypofibrinogenemia is not common in sepsis, ISTH has previously confirmed the usefulness of this simple score, as it has developed SIC criteria to guide anticoagulant therapy [35]. In a study conducted by Tang et al. [38], it was shown that routine prophylactic use of heparin in patients with SIC score $>4$ and D-Dimer $>3.0 \mathrm{mg} / \mathrm{L}$ decreased mortality.

\section{RADIOLOGY}

In patients with COVID-19 infection, atypical findings were observed along with typical findings such as peripheral ground glass, consolidation in thorax CT $[39,40]$. Infected areas related to PE can be seen in thorax CT. In some patients, enlarged subsegmental pulmonary vessels can be seen [41, 42]. Dual energy CT imaging can be used to detect lung perfusion defects. Although PE has not been observed in many COVID-19 infections, striking perfusion abnormalities that have not been previously identified have been recorded [39].

In patients with COVID-19 pneumonia, increased perfusion in the areas of lung opacity and proximal lung areas and decreased perfusion in the peripheral lung areas were observed. This may be due to the relative insufficiency of physiological hypoxic pulmonary vasoconstriction and pulmonary vascular dilatation due to excessive activation of inflammation. Perfusion abnormalities suggest an intrapulmonary shunt towards regions where gas exchange is impaired with pulmonary vascular dilatation. As a result, ventilation-perfusion mismatch worsens and hypoxia develops.

\section{TREATMENT}

\section{Treatment Indications}

The most important treatment for all coagulopathies is the treatment of the underlying condition. Patients with VTE, inpatient medical, surgical, and COVID-19 therapy should receive anticoagulant therapy unless there is a contraindication to anticoagulation (active bleeding or severe bleeding within the previous 24 to 48 hours). All patients with COVID-19 should receive thromboprophylaxis, especially in the intensive care unit. Even in the absence of VTE, it would be appropriate to use moderate or therapeutic dose anticoagulation in severely ill patients. Some people without VTE should also receive prolonged thromboprophylaxis after discharge from the hospital. People with other risk factors such as immobilization, surgery, or trauma should use prophylactic anticoagulation after discharge. The risk of bleeding needs to be included in the decision-making process. In patients with COVID-19 who do not require hospitalization, thromboprophylaxis treatment may also be appropriate, especially for those with previous VTE or other thrombotic risk factors such as recent surgery, trauma or immobilization. It will be more correct for the clinician to make this decision. There is no study ad-

\section{Table 5. Anticoagulant therapy in COVID-19 patients}

1. High suspicion of acute thromboembolism- AMI, acute massive PE, DVT in extremity, arterial thrombosis- Thrombolytic therapy, anticoagulant should be given in therapeutic dose.

2. High suspicion of acute thromboembolism- AMI, acute massive PE, DVT in extremity, no arterial thrombosis- anticoagulant should be given at therapeutic dose.

3. Suspected acute thromboembolism can be low-anticoagulant therapy, patient-anticoagulant therapy continued or short-acting parenteral drugs can be switched.

4. Low suspicion of acute thromboembolism - patient not receiving anticoagulant treatment - being treated in hospital intensive care unit - anticoagulant (prophylactic, at therapeutic dose).

5. Low suspicion of acute thromboembolism - patient not receiving anticoagulant therapy - receiving treatment in hospital ward - prophylactic LMWH, Unfractionated heparin, fondaparinux.

6. Low suspicion of acute thromboembolism - patient not receiving anticoagulant treatment - not receiving hospital treatment - prophylactic anticoagulation should be given if the risky group.

$\mathrm{AMI}=$ acute myocardial infarction, DVT = deep venous thrombolism, LMWH = low molecular weight heparin, $\mathrm{PE}=$ pulmonary embolism 


\section{Table 6. Coagulopathy treatment in COVID-19 patients}

1. Thrombosis prophylaxis in patients with 1 st D-dimer $<1000 \mathrm{ng} / \mathrm{ml}$ a- $\mathrm{CrCl}>30 \mathrm{ml} / \mathrm{min}, \mathrm{BMI}<40 \mathrm{~kg} / \mathrm{m}^{2}$ : Enoxaparin $40 \mathrm{mg} /$ day.

BMI $>40 \mathrm{~kg} / \mathrm{m}^{2}$ : Enoxaparin $40 \mathrm{mg} 2 \times 1 \mathrm{SC}$, BMI $<40 \mathrm{~kg} / \mathrm{m}^{2}$ : Enoxaparin $40 \mathrm{mg} /$ day SC b- $\mathrm{CrCl}<30 \mathrm{ml} / \mathrm{min}$ : Standard heparin $5000 \mathrm{U}$ SC $2 \times 1$ or $3 \times 1$ or Dose reduced low molecular weight heparin is recommended.

2. D-Dimer $>1000 \mathrm{ng} / \mathrm{ml}$ or patients with severe illness

Enoxaparin: $0.5 \mathrm{mg} / \mathrm{kg} \mathrm{SC}$ every 12 hours.

a- $\mathrm{CrCl}<30 \mathrm{ml} / \mathrm{min}$ : Standard heparin $5000 \mathrm{U} \mathrm{SC} 2 \times 1$ or $3 \times 1$ or dose reduced low molecular weight heparin recommended.

3. Patients with a previous history of atrial thrombosis or venous thrombosis

(No change if taking more than 90 days, heparin in therapeutic dose is recommended if taking it for less than 90 days).

$\mathrm{BMI}=$ body mass index, $\mathrm{CrCl}=$ creatinine clearance, $\mathrm{SC}=$ subcutaneous

dressing thromboprophylaxis for patients in this category with COVID-19. Anticoagulant treatment in COVID-19 patients is summarized in Table 5 and Table 6 [43]. LMWH is the basis of treatment. However, heparin treatment should be avoided in cases where there are contraindications such as active bleeding, thrombocytopenia, HIT and heparin-induced allergy. In these cases, an alternative agent such as fondaparinux can be used.

\section{Treatment Time}

The duration of heparin prophylaxis should be decided based on the patient's thrombosis risk, mobilization, and returning of inflammation markers to normal levels. Individuals with documented VTE should receive anticoagulation therapy for at least three months. Among the post-discharge prophylaxis options, there are studies suggesting the use of $10 \mathrm{mg}$ rivaroxaban daily for 31-39 days [4].

\section{Treatment Success}

In evaluating the effectiveness of heparin therapy, aPTT, ACT, AntiFXa, AT-3 and platelet levels should be monitored. A significantly better prognosis was observed with LMWH and anticoagulant therapy in COVID-19 patients [44]. In a retrospective study of 449 individuals with severe COVID-19, enoxaparin (40-60 mg once a day) was found to be associated with improved survival, especially in those with high DDimer [38]. In addition to its anticoagulant effect, it is thought that heparin binds inflammatory cytokines, in- hibits neutrophil chemotaxis and leukocyte migration, neutralizes positively charged peptide $\mathrm{C} 5 \mathrm{a}$ and sequesters acute phase proteins $[2,44,45]$.

\section{Treatment Failure}

The prevalence of venous thromboembolism (VTE) is increasing, especially in critically ill individuals, despite prophylactic anticoagulation. The study by Klok et al. [6] showed that the use of LMWH prophylactic at low doses did not prevent thrombotic complications. In addition, it has been shown that the cumulative incidence of thrombotic complications in COVID-19 patients admitted to the intensive care unit is extremely high (>30\%) [6]. Despite the use of prophylactic anticoagulation, VTE, DVT and APE can be seen in one third of patients in the intensive care unit with COVID-19. In a study conducted, it was observed that DVT developed in $14 \%$ of 184 patients who received standard dose thromboprophylaxis in the intensive care unit due to COVID-19 infection [6]. Some experts have recommended more aggressive thromboprophylaxis dosage for the risk of VTE [43].

\section{Plasminogen Activator (tPA)}

Tissue plasminogen activator (tPA) is appropriate unless there is contraindication in cases of extremitythreatening DVT, massive APE, acute stroke and acute myocardial infarction.

\section{Dipyridamole}

Dipyridamole use may have anti-inflammatory, 
antiaggregant and possible antiviral efficacy. However, there is no definitive recommendation for COVID-19 disease. Thrombosis prophylaxis should be administered to all COVID-19 patients and antiaggregant patients should be administered to those approved by the physician [36].

\section{Mechanical Thrombosis Prophylaxis}

In patients for whom pharmacological prophylaxis is contraindicated, mechanical thrombosis prophylaxis with intermittent pneumatic compression devices or compression stockings is recommended. Mechanical prophylaxis can be applied in addition to standard pharmacological prophylaxis in immobile patients.

\section{Bleeding}

Bleeding is less common in patients with COVID19. However, it may occur especially in patients receiving anticoagulation or due to trauma. The bleeding approach is similar to people without COVID-19. Withdrawal of anticoagulant therapy may include specific treatments such as transfusions or factor replacement for thrombocytopenia or hypofibrinogenemia. Antifibrinolytic agents (tranexamic acid, epsilon aminocaproic acid) are generally not used in patients with DIC due to the concern that they may disrupt the balance against thrombosis. Fibrinogen is frequently increased in COVID-19. If there is no bleeding attributable to hypofibrinogenemia or dysfibrinogenemia (fibrinogen activity level $<150-200 \mathrm{mg} / \mathrm{dL}$ ), fibrinogen supplementation is not required. Platelet suspension in a patient with major bleeding coagulopathy or DIC if the platelet count is $<50 \times 109 / \mathrm{L}$; If PT and / or aPTT prolongation, INR $>1.8$, fresh frozen plasma and fibrinogen concentrate or cryoprecipitate can be replaced if fibrinogen level is $<1.5 \mathrm{~g} / \mathrm{L}$. Protein complex concentrate is not recommended as recombinant FVIIa is unknown.

\section{CONCLUSION}

Coronavirus disease-19 (COVID-19) is a potentially fatal disease. SARS-COV2 can cause DIC. In addition, COVID-19 infection can also cause venous and / or arterial thrombosis, including APE, AMI, limb thrombosis, and central nervous system. When monitoring COVID-19 patients, platelet, PT and aPTT, fib- rinogen and D-dimer monitoring should be initiated every 1-2 days, especially in critically ill patients. High D-Dimer levels are associated with high mortality; may be a marker of infection / sepsis, cytokine storm, and impending organ failure. DIC can be seen in COVID-19 patients. However, unlike DIC, fibrinogen is usually high. Clotting times and platelet counts are usually normal. Therefore, it is appropriate to use SIC criteria in the follow-up of COVID-19 patients. Treatment of the underlying disease is the most important treatment for all coagulopathies. A significantly better prognosis was observed in patients treated with LMWH anticoagulants. Bleeding is less common than clotting in patients with COVID-19. However, it may occur in patients receiving anticoagulation or due to trauma. The bleeding approach is similar to people without COVID-19.

\section{Authors' Contribution}

Study Conception: HÖ, MT, EÜ, MD; Study Design: HÖ, MT, EÜ, MD; Supervision: HÖ, MT, EÜ, MD; Funding: HÖ, MT, EÜ, MD; Materials: HÖ, MT, EÜ, MD; Data Collection and/or Processing: HÖ, MT, EÜ, MD; Statistical Analysis and/or Data Interpretation: HÖ, MT, EÜ, MD; Literature Review: HÖ, MT, EÜ, MD, NAI; Manuscript Preparation: HÖ, MT, EÜ, MD, NAI and Critical Review: HÖ, MT, EÜ, MD, NAI.

\section{Conflict of interest}

The authors disclosed no conflict of interest during the preparation or publication of this manuscript.

\section{Financing}

The authors disclosed that they did not receive any grant during the conduction or writing of this study.

\section{REFERENCES}

1. Chen N, Zhou M, Dong X, Qu J, Gong F, Han Y, et al. Epidemiological and clinical characteristics of 99 cases of 2019 novel coronavirus pneumonia in Wuhan, China: a descriptive study. Lancet 2020;395:507-13.

2. Huang C, Wang Y, Li X, Ren L, Zhao J, Hu Y, et al. Clinical features of patients infected with 2019 novel coronavirus in Wuhan, China. Lancet 2020;395:497-506.

3. Tang N, Li D, Wang X, Sun Z. Abnormal coagulation parameters are associated with poor prognosis in patients with novel coronavirus pneumonia. J Thromb Haemost 2020;18:844-7. 
4. https://www.hematology.org/COVID-19/COVID-19-and-coagulopathy (Accessed on April 20, 2020).

5. Connors JM, Levy JH.. Thromboinflammation and the hypercoagulability of COVID-19. J Thromb Haemost 2020;18;155961.

6. Klok FA, Kruip MJHA, Van Der Meer NJM, Arbous MS, Gommers DAMPJ, Kant KM, et al. Confirmation of the high cumulative incidence of thrombotic complications in critically ill ICU patients with COVID-19: an updated analysis. Thromb Res 2020;191:148-50.

7. Ciceri F, Beretta L, Scandroglio AM, Colombo S, Landoni G, Ruggeri A, et al. Microvascular COVID-19 lung vessels obstructive thromboinflammatory syndrome (MicroCLOTS): an atypical acute respiratory distress syndrome working hypothesis. Crit Care Resusc 2020;22:95-7.

8. Krishna S, Eswaran N. Recent research progress and current understanding of severe acute respiratory syndrome coronavirus 2 (SARS-CoV-2). Asian J Biol 2020;10:9-21.

9. Schmaier AH. The plasma kallikrein-kinin system counterbalances the renin-angiotensin system. J Clin Invest 2002;109:10079.

10. Van de Veerdonk F, Netea MG, van Deuren M, van der Meer JW, de Mast Q, Bruggemann R, et al. Kinins and cytokines in COVID-19: a comprehensive pathophysiological approach. Preprints 2020;2020040023

11. Panigada M, Bottino N, Tagliabue P, Grasselli G, Novembrino C, Chantarangkul V, et al. Hypercoagulability of COVID-19 patients in intensive care unit. A report of thromboelastography findings and other parameters of hemostasis. J Thromb Haemost 2020;18:1738-42.

12. Ranucci M, Ballotta A, Di Dedda U, Bayshnikova E, Dei Poli M, Resta M, et al. The procoagulant pattern of patients with COVID-19 acute respiratory distress syndrome. J Thromb Haemost 2020;18:1747-51.

13. https://www.esicm.org/wp-content/uploads/2020/04/863_author_proof.pdf (Accessed on April 25, 2020.

14. Bowles L, Platton S, Yartey N, Dave M, Lee K, Hart DP, et al. Lupus anticoagulant and abnormal coagulation tests in patients with Covid-19. N Engl J Med 2020;383:288-90.

15. Zhou Y, Fu B, Zheng X, Wang D, Zhao C, Qi Y, et al. Pathogenic $\mathrm{T}$ cells and inflammatory monocytes incite inflammatory stroms in severe COVID-19 patients. Nat Sci Rev 2020;6:9981002 .

16. Magro C, Mulvey JJ, Berlin D, Nuovo G, Salvatore S, Harp $\mathrm{J}$, et al. Complement associated microvascular injury and thrombosis in the pathogenesis of severe COVID-19 infection: a report of five cases. Transl Res 2020;220:1-3.

17. Singer M, Deutschman CS, Seymour CW, Shankar-Hari M, Annane D, Bauer M, et al. The third international consensus definitions for sepsis and septic shock (Sepsis-3). JAMA 2016;315:801-10.

18. Levi M, van der Poll T. Coagulation and sepsis. Thromb Res 2017;149:38-44.

19. Schmitt FCF, Manolov V, Morgenstern J, Fleming T, Heitmeier S, Uhle F, et al. Acute fibrinolysis shutdown occurs early in septic shock and is associated with increased morbidity and mortality: results of an observational pilot study. Ann Intens Care
2019;9:19.

20. Monteleone G, Sarzi-Puttini PC, Ardizzone S. Preventing COVID-19-induced pneumonia with anticytokine therapy. Lancet Rheumatol 2020;2:e255-6.

21. Mastellos DC, Ricklin D, Lambris JD. Clinical promise of next-generation complement therapeutics. Nat Rev Drug Discov 2019; 18:707-29.

22. Thompson BT, Chambers RC, Liu KD. Acute respiratory distress syndrome. N Engl J Med 2017;377:562-72.

23. Tan CW, Low JGH, Wong WH, Chua YY, Goh SL, Ng HJ. Critically ill COVID-19 infected patients exhibit increased clot waveform analysis parameters consistent with hypercoagulability. Am J Hematol 2020;95:E156-8.

24. Gattinoni L, Coppola S, Cressoni M, Busana M, Rossi S, Chiumello D. Covid-19 does not lead to a "typical" acute respiratory distress syndrome. Am J Respir Crit Care Med 2020;201:1299300 .

25. Gupta N, Zhao YY, Evans CE. The stimulation of thrombosis by hypoxia. Thromb Res 2019;181:77-83.

26. Begbie M, Notley C, Tinlin S, Sawyer L, Lillicrap D. The factor VIII acute phase response requires the participation of NFאB and C/EBP. Thromb Haemost 2000;84:216-22.

27. Wang D, Hu B, Hu C, Zhu F, Liu X, Zhang J, et al. Clinical characteristics of 138 hospitalized patients with 2019 novel coronavirus-infected pneumonia in Wuhan, China. JAMA 2020;323:1061-9.

28. Fox SE, Akmatbekov A, Harbert JL, Li G, Brown JQ, Vander Heide RS. Pulmonary and cardiac pathology in African American patients with COVID-19: an autopsy series from New Orleans. Lancet Respir Med 2020;8:681-6.

29. Memikoğlu O, Genç V. COVİD-19. E-Kitap, Ankara Üniversitesi Basımevi: Ankara, 2020.

30. Bellosta R, Luzzani, L, Natalini G, Pegorer MA, Attisani L, Cossu LG, et al. Acute limb ischemia in patients with COVID19 pneumonia. J Vasc Surg 2020;72:1864-72.

31. Taylor FB, Toh CH, Hoots WK, Wada H, Levi M. Towards definition, clinical and laboratory criteria, and a scoring system for disseminated intravascular coagulation. Thromb Haemost 2001;86:1327-30.

32. Thachil J, Tang N, Gando S, Falanga A, Cattaneo M, Levi M, et al. ISTH interim guidance on recognition and management of coagulopathy in COVID-19. J Thromb Haemost 2020;18:10236.

33. Levi M, Toh CH, Thachil J, Watson HG. Guidelines for the diagnosis and management of disseminated intravascular coagulation. British Committee for Standards in Haematology. Br J Haematol 2009;145:24-33.

34. Di Nisio M, Baudo F, Cosmi B, D'Angelo A, De Gasperi, A, Malato A, et al. Diagnosis and treatment of disseminated intravascular coagulation: guidelines of the Italian Society for Haemostasis and Thrombosis (SISET). Thromb Res 2012;129:e177-84.

35. Iba T, Di Nisio M, Levy JH, Kitamura N, Thachil J. New criteria for sepsis-induced coagulopathy (SIC) following the revised sepsis definition: a retrospective analysis of a nationwide survey. BMJ Open 2017;7:e017046.

36. Turkey Ministry of Health, General Directorate of Public 
Health, COVID-19 (SARS-CoV-2 Infection) Guide. April 12, 2020, pp. 64-8.

37. Iba T, Levy JH, Warkentin TE, Thachil J, van der Poll T, Levi M; Scientific and Standardization Committee on DIC, and the Scientific and Standardization Committee on Perioperative and Critical Care of the International Society on Thrombosis and Haemostasis. Diagnosis and management of sepsis-induced coagulopathy and disseminated intravascular coagulation. J Thromb Haemost 2019;17:1989-94.

38. Tang N, Bai H, Chen X, Gong J, Li D, Sun Z. Anticoagulant treatment is associated with decreased mortality in severe coronavirus disease 2019 patients with coagulopathy. J Thromb Haemost 2020;18:1094-9.

39. Chung M, Bernheim A, Mei X, Zhang N, Huang M, Zeng X, et al. CT imaging features of 2019 novel coronavirus (2019nCoV). Radiology 2020;295:202-7.

40. Pan F, Ye T, Sun P, Gui S, Liang B, Li L, et al. Time course of lung changes on chest CT during recovery from 2019 novel coronavirus (COVID-19) pneumonia. Radiology 2020;295:715-
21.

41. Bai HX, Hsieh B, Xiong Z, Halsey K, Choi JW, Tran TML, et al. Performance of radiologists in differentiating COVID-19 from viral pneumonia on chest CT. Radiology 2020;296:E46-54. 42. Caruso D, Zerunian M, Polici M, Pucciarelli F, Polidori T, Rucci C, et al. Chest CT features of COVID-19 in Rome, Italy. Radiology 2020;296:E79-85.

43. Cuker A, Peyvandi F. Coronavirus disease 2019 (COVID19): Hypercoagulability. UpToDate 2020. https:/www.uptodate.com/contents/coronavirus-disease-2019covid-19-hypercoagulability (Accessed on November 26, 2020). 44. Guan WJ, Ni ZY, Hu Y, Liang WH, Ou CQ, He JX, et al; China Medical Treatment Expert Group for Covid-19. Clinical characteristics of coronavirus disease 2019 in China. N Engl J Med 2020;382:1708-20.

45. Poterucha TJ, Libby P, Goldhaber SZ. More than an anticoagulant: Do heparins have direct anti-inflammatory effects? Thromb Haemost 2017;117:437-44. 